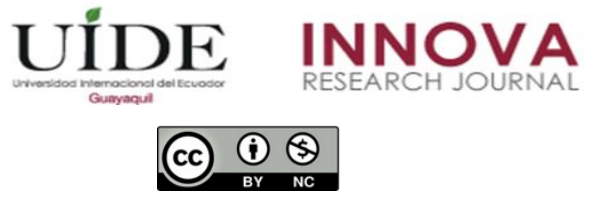

INNOVA Research Journal, ISSN 2477-9024

(Enero-Abril 2021). Vol. 6, No.1 pp. 195-215

DOI: https://doi.org/10.33890/innova.v6.n1.2021.1522

URL: http://revistas.uide.edu.ec/index.php/innova/index

Correo: innova@uide.edu.ec

\title{
Aplicación de algoritmos etnomatemáticos en el aprendizaje significativo de estudiantes universitarios
}

\section{The application of ethnomathematical algorithms in the meaningful learning of university students}

Ronald Jesús Alarcón Anco

\author{
Di https://orcid.org/0000-0002-7971-5302 \\ Universidad Norbert Wiener, Perú \\ Heyden Neil Flores de la Cruz \\ (D) https://orcid.org/0000-0003-4993-3429 \\ Investigador Independiente, Perú
}

Autor para correspondencia: ronaldalarcon240277@gmail.com; ecoisp@ hotmail.com

Fecha de recepción: 05 de septiembre de 2020 - Fecha de aceptación: 02 de diciembre de 2020

\begin{abstract}
Resumen
El artículo presenta resultados del impacto de los algoritmos etnomatemáticos en el aprendizaje significativo de la matemática en los estudiantes universitarios en 2019. El estudio persiguió el propósito de determinar como la aplicación de los algoritmos etnomatemáticos afecta el aprendizaje significativo de la matemática. En él se verifica la aplicación frecuente de dichos algoritmos en las sesiones de aprendizaje puede mejorar significativamente el aprendizaje de la matemática, en estudiantes universitarios. Se aplicaron tres técnicas: observación, cuestionario y una ficha de entrevista aplicada a 25 estudiantes universitarios, aplicado en dos pruebas, una prueba preliminar y una prueba de cierre. Con resultados positivos, mostrados a través de los valores del estadístico $\mathrm{T}=1.02$ (primera hipótesis operacional) por lo que se acepta Hi y se rechaza Ho. Y en el estadístico, T = 0,09(tercera hipótesis operacional), que está en el área de aceptación, para lo cual aceptamos Hi y rechazamos Ho. Está comprobado estadísticamente que el uso de algoritmos etnomatemáticos influye en el aprendizaje significativo de los estudiantes universitarios. Concluyéndose que los estudiantes universitarios desarrollan mejor sus habilidades lógicas y matemáticas utilizando algoritmos etnomatemáticos. Se deben profundizar estudios sobre el efecto que produce la aplicación de la etnomatemática como estrategia de enseñanza aprendizaje en la formación inicial docente a nivel de Escuelas Superiores y Universidades.
\end{abstract}

Palabras claves: etnomatemática; aprendizaje significativo; actividades cognoscitivas; aprendizaje por representaciones; aprendizaje de proposiciones.

\footnotetext{
Abstract

The article presents results of the impact of ethnomathematical algorithms on the meaningful learning of mathematics in university students in 2019. The study pursued the purpose of determining how the application of ethnomathematical algorithms affects the meaningful learning
} 
of mathematics. In it, the frequent application of these algorithms in the learning sessions can significantly improve the learning of mathematics in university students. Three techniques were applied: observation, questionnaire and an interview sheet applied to 25 university students, applied in two tests, a preliminary test and a closing test. With positive results, shown through the values of the statistic $\mathrm{T}=1.02$ (first operational hypothesis) so that $\mathrm{Hi}$ is accepted and $\mathrm{Ho}$ is rejected. And in the statistic, $\mathrm{T}=0.09$ (third operational hypothesis), which is in the acceptance area, for which we accept $\mathrm{Hi}$ and reject Ho. It is statistically proven that the use of ethnomathematical algorithms influences the meaningful learning of university students. Concluding that university students better develop their logical and mathematical skills using ethnomathematical algorithms. Studies should be deepened on the effect produced by the application of ethnomathematics as a teaching-learning strategy in initial teacher training at the level of Higher Schools and Universities.

Keywords: Ethnomathematics; significant learning; cognitive activities; learning by representations; learning propositions.

\section{Introducción}

La aplicación de la etnomatemática, ha hecho que muchos estudiantes universitarios a partir de su cotidianidad, en el uso de operaciones aritméticas, mejoren significativamente su capacidad para realizar operaciones matemáticas.

Según el profesor; D'Ambrosio, (2001) describe que las acciones que desarrolla las matemáticas dentro la sociedad son aplicadas según su cultura étnica, lo cual los lleva a simbolizar y crear nomenclaturas según sus propias necesidades. Lo que Valencia y Díaz (2019) afirman actualmente el valor del contexto circundante al aula, en el que realiza sus vivencias el estudiante, se reconocen los saberes extraescolares, se logra una vinculación de los objetos matemáticos aprehendidos en el aula y su vida cotidiana. Usar el término "etnomatemático" es dar referencia a uso no estructurado de las propias matemáticas, tiene más un uso específico según la propia sociedad que la práctica, por ejemplo las comunidades indígenas la usan en pequeñas escalas, por medio del trueque, símbolos, etc.

Según Gerdes (1989) manifiesta una perspectiva educacional de la etnomatemática, como una reivindicación de la cultura primigenia de los pueblos logrando su aprendizaje usando prácticas numerativas creadas por su sobrevivencia, se intenta lograr objetivos sociales, por medio de su cultura y lo cual persisten con su aprendizaje aplicando esta matemática incipiente que es común para su aprendizaje básico, que en otras sociedades es excluida.

Según Fuentes (2019) nombra a la etnomatemática como una perspectiva sociocultural, la cual da énfasis a la creatividad y la crítica, estableciendo el papel del docente como facilitador lo que permite que la práctica pedagógica tenga su propio espacio ante un currículo definido a priori, y tomando las propuesta de Saca (2019) que dicen la sabiduría ancestral trata del encuentro e interacción de la gran familia humana, en tanto los seres vivos somos parte de una familia, por ello se materializa el acto de escuchar, valorar y aprender de diversos saberes. Ascher (1982) nombra a la etnomatemática como una matemática oriundo de los pueblos iletrados, y tomando las propuesta de Bishop (2005) que dicen la cultura social y las 
matemática hacen una etnomatemática. Finalmente la etimología que presenta D'Ambrosio (1985) ampliando el prefijo "etno" entramos a grupos sociales comunitarias tradicionalistas en sus culturas y sus creencias, lo cual por su diversidad de entendimiento desarrollan actividades comunes de su propia sociedad, razón por la cual también practican la etnomatemática para poder vivir, así controlan sus necesidades básicas. Finalmente la etimología que presenta Aroca (2016) implica que la Etnomatemática se presenta como las tic de la de mathema en una etno, lo que obstaculiza su interpretación e incluso considera que es un reduccionismo ya que lo lingüístico en la enseñanza y aprendizaje de las matemáticas es interpretada a partir de las tics de mathema en una etno. Una de la razones fundamentales de la aplicación del estudio está en revalorar la diversidad cultural del uso de la matemáticas y cómo éstas a través del tiempo se han mantenido muy vigentes sin medir condiciones, capacidades, clases, y lo que queremos es justamente hacer que gracias a la etnomatemática se pueda aplicar nuevos conocimiento a la educación superior que son de mucha utilidad a la sociedad universitaria.

Partiendo de la premisa de que muchos estudiantes universitarios desconocen los diferentes procesos de algoritmos etnomatemáticos, con lo cual consiguen desarrollar actividades comunes, para poder sobrevivir en su vida diaria; no se dan cuenta que están adquiriendo un aprendizaje significativo, imposibilitando una autoevaluación, lo que llamamos pedagógicamente metacognición, este conocimiento muchas veces no demuestra el aprendizaje y amor a la matemática, lo cual siempre genera reticencia a su aprendizaje lo cual los lleva a un bajo rendimiento en matemáticas. Tal como manifiesta Ascher y D'Ambrosio (1994) se menciona, que para Gerdes (1996) la etnomatemática tiene un trabajo de reconsideración del uso de la matemáticas, usando modelos pedagógicos y cognitivos en su aprendizaje en las personas. Demostrando que cada estudiante universitario logra adaptar su matemática para poder aplicarla dentro su formación profesional, recurriendo muchas veces a su cultura de formación o vida social.

Según Viteri (2015) manifiesta una perspectiva educacional de la etnomatemática, se obtiene al problematizar el hecho de que cuente con el conocimiento de los saberes ancestrales, ya que ello permitiría desarrollar investigaciones participativas, en las que la identidad cultural convierta, a los estudiantes, en actores protagónicos de los procesos pedagógicos. Partiendo de la premisa de que muchos estudiantes universitarios desconocen los diferentes procesos de algoritmos etnomatemáticos, con lo cual consiguen desarrollar actividades comunes, para poder sobrevivir en su vida diaria; no se dan cuenta que están adquiriendo un aprendizaje significativo, imposibilitando una autoevaluación, lo que llamamos pedagógicamente metacognición, este conocimiento muchas veces no demuestra el aprendizaje y amor a la matemática, lo cual siempre genera reticencia a su aprendizaje lo cual los lleva a un bajo rendimiento en matemáticas. Tal como manifiesta Bonilla, Allccahuallpa, Rosa y Reyes (2018) de igual manera lo manifiesta Ascher y D'Ambrosio (1994) se menciona, que para Gerdes (1996) la etnomatemática tiene un trabajo de reconsideración del uso de la matemáticas, usando modelos pedagógicos y cognitivos en su aprendizaje en las personas. Se torna difícil incorporar la matemática ancestral a los programas interculturales, a fin de lograr ello hay que cambiar los paradigmas al interior de las instituciones educativas y tomar en consideración la cosmovisión, por ello es necesario que se articule la escuela con las comunidades indígenas, ello permitirá vencer la concepción eurocéntrica de la matemática que se resiste a la interculturalidad. 
Esto (escaso interés) conduce a la apatía, al automatismo, a un aprendizaje sin significado para el universitario, impidiendo además que el estudiante sea consciente de sus diferentes habilidades y capacidades y que pueda ir descubriendo en el proceso educativo y laboral su vocación personal y social. Por ello se formuló el problema: ¿Qué efecto produce la aplicación de algoritmos etnomatemáticos en el aprendizaje significativo de la matemática? , el mismo que se ha investigado en razón de que a nivel Universitario se percibe que la matemática no motiva a la mayoría de estudiantes, son pocos que se interesan por desarrollarla, lo que se agudiza cuando se intenta partir de sus experiencias cotidianas o de su identidad histórica como fuente para analizar la matemática. Este problema afecta la capacidad reflexiva del docente, lo cual se percibe cuando desarrolla su actividad en las zonas rurales, en las que carece de los elementos propias de la urbanidad y encuentra que se le hace más difícil facilitar el aprendizaje de la matemática, generando estudiantes desinteresados en esta ciencia con las ulteriores consecuencias en su futuro como ciudadano y/o profesional.

En ese sentido este articulo intenta en principio teorizar este problema, para ir modificando patrones conductuales y didácticos, que vayan haciendo posible el gran cambio.

El profesor D’Ambrosio (2002) considera que la etnomatemática tiene base en la historia y filosofía de la matemática, incluyendo a la pedagógicas, por su dinamismo epistemológico, político y educacional. Por lo tanto la etnomatemática es una disciplina de la matemática en la educación, por lo que se está enriqueciendo, por diferentes campos, como el filosófico, el antropológico, el histórico; de la misma manera; Villota y Singo (2018) considera que la etnomatemática se funda en una episteme con profundas raíces en los pueblos aborígenes de América latina, por lo que su ethos al medir la dimensionalidad tiempo-espacio en contextos comparables con la matemática de origen europeo, árabe, babilonio y egipcio.

El docente intercultural tiene que utilizar estrategias que estén articuladas con las necesidades y expectativas de sus estudiantes las que debe aplicar en la enseñanza de la matemática, siguiendo a Cahuana, Salinas y Ruiz (2018) se afirma que así se puede lograr que esta ciencia sea interesante y no aburrida no cause temor; y así poder cumplir con el objetivo de evaluar en qué medida la etnomatemática puede incidir en trazar planes para resolver problemas.

Las autoras Knijnik, Wanderer, Giongo, y Duarte (2012) dicen que la etnomatemática es un campo de acción en el aprendizaje rural educativo en las comunidades, lo cual se asocian al uso de una matemática escolar no muy entendida por estas comunidades. Y según Miarka y Viggiani (2012) en su tesis doctoral muestran en caracterizar y clasificar la etnomatemática, como el comportamiento de una sociedad siempre que se refleja por un conjunto de disciplinas y esto conlleva a utilizar una etnomatemática y ethnogeometría como parte esencial de la matemática en mejoras de una educación multicultural de nuestros estudiantes universitarios, por lo tanto este dominio etnomatemático refleja como un medio de vivir y adquirir nuevos conocimiento y mejoras en el aprendizaje significativo de la matemática en nuestro sociedad. Las matemáticas desde nuestros antepasados hasta la actualidad, siempre están presente y la forma de aplicarla es a través de algoritmos etnomatemáticos, por su necesidad diaria de las diferentes sociedades que emergen en nuestra vida. 
Según el Prof. D'Ambrosio, (2001) trata mucho a la etnomatemática por su existencia en grupo culturales arraigados en sociedad ancestral y actual y todas sus actividades sociales los conllevan a tener actividades donde muchas de ellas requieren de soluciones matemáticas y en su propia necesidad desarrollan un algoritmo matemático para solucionar ese problema social cotidiano, lo cual se conocerá como etnomatemática. Gómez (2019) estima que es posible asimilar que la matemática es propia de un contexto sociocultural, por lo que debe ser definida y comprendida como parte del accionar pedagógico. De la misma manera. Barton. (1997) Manifiesta que la matemática es parte de la etnomatemática, entonces para aprender Matemática indiscutiblemente se debe pasar por la etnomatemática. Los docentes no han tomado en cuenta que, en el contexto sociocultural, se halla la auténtica manera de aprender matemática.

Según, D’Ambrosio (1985), manifiesta que las formas de aplicar la matemática en diferentes grupos culturales son propias de cada una de ellas, que en su propio entendimiento se le puede conocer como etnomatemática. Por tanto los modos de aplicar su propia matemática, estos grupos culturales desarrollan algoritmos propios de ellos lo cual vienen hacer aprendizaje etnomatemático. Este algoritmo que, se desarrolla en la cotidianeidad puede ser interpretado y llevado al interior del aula con fines pedagógicos a fin de vencer, el carácter condicionante de las interacciones interculturales que de acuerdo a Dos Santos y Santos (2018) develan relaciones de poder asimétricas, más o menos intensas, en ese contexto logran una variedad de formas de integración como la autonomía, autodeterminación y conquista la misma que deviene en la colonización. Por lo que existe un carácter político en lo intercultural y lo que, sin duda, se refleja en la práctica educativa intercultural.

Para comprender la etimología de la etnomatemática. D'Ambrosio (1985). Tiene su concepción de: etno+matema+tica, donde: etno=, entorno natural y cultural; y matema= explicar, enseñar, comprender, manejar, lidiar; y ticas= las artes, técnicas, maneras, estilos. Por tanto se conceptualiza que, "etno" es el "entorno natural y cultural" en donde el hombre en sus diferentes etapas de vida se ha condicionado a sobrevivir desarrollando actividades con un accionar de las cosas como se le presenta, alimentarse, descansar y todas esa actividades son cambiantes por las noches y días, y semanas y meses, y genere hacer sumatoria de cada momento de su vida. Y matema "las artes, técnicas, maneras, estilos " Refiriéndose a las formas de expresión y desarrollo mental, a través de la realidad mediante un métodos gráfico, simbólico, petroglífico, propio de su cultura social. Y finalmente "ticas" es el uso metodológico de cómo llegar, al individuo, desarrollando un proceso de compartir las experiencias aprendidas entre personas que tienen el interés a poseer este conocimiento etnomatemático, el cual ayudara a solucionar problemas socio-culturales en diferentes sociedades que emergen por aprender y condicionarse a la vida.

El profesor D’ Ambrosio, (2001) dice que la Matemática es una parte de la etnomatemática, entonces la etnomatemática es primigenia a la matemática, siendo el primer aprendizaje de toda sociedad arraigada a una cultura de aprendizaje social. Santana (2019) afirma que la vida diaria del estudiante es una expresión de la interacción cotidiana respecto a las matemáticas y presumiblemente por lo que su conocimiento matemático debe ser reconocido. Según Barton, (1997) teniendo un interés más de aprendizaje social mediante su propia cultura del individuo, mejora otros conocimientos algorítmicos. Ello debido a la necesaria contextualización del hacer y aprender 
matemáticas, respetando lo que el estudiante considera matemático. Donde el Profesor D'Ambrosio, (2001) la ubica en una dimensión socio-antropológica, nombrándolos como: la matemática viene hacer el concepto de las prácticas; el matemático viene hacer el actor de las prácticas, siendo el grupo interesado en aprender usando sus propios medios sociales, culturales, que claramente comprende la manera como se matematiza, es decir: es la forma como ellos desarrollan actividades algorítmicas a través de la etnomatemática, lo cual no es descriptible con exactitud pero si ayuda mucho a desarrollar nuevos algoritmos para el aprendizaje de la matemática. En este sentido el individuo se inserta en su contexto y en el desarrolla matemática como parte de su cotidianeidad, ello configura la etnomamatemática.

Para Guzmán (2018) realizar un reconocimiento de la diversidad y el multiculturalismo de la sociedad implica que el fenómeno educativo se transforme, redireccione y resignifique su papel en relación con la identidad de la persona y su contexto, a fin de construir su proyecto personal de vida, y así aportar en la transformación socio-cultural de la comunidad, sin perder de vista el hecho de pertenecer a una sociedad globalizada. Siendo la matemática un vehículo de esta resignificación y sobre todo la practica pedagógica etnomatemática la que logre esta amalgama de identidad entre lo local y lo global como componentes del contexto.

Blanco-Álvarez (2006) (2011) reconstruye a la etnomatemática, mediante categorías:

- $\quad$ El uso de una matemática incipiente en estratos sociales y comunidades "iletradas". Tienen su propio algoritmo de uso, esto conlleva a tener una matemática básica.

- $\quad$ El uso de la matemática fundamental básica en comunidades indígenas y ancestrales: Donde cada comunidad desarrolla una matemática según sus pensamientos e ideas de vida.

- $\quad$ El uso de artículos de su propia cultura indígena como herramientas pedagógicas para la enseñanza de la matemática occidental: Muchas de estas culturas usan herramientas como los kipus para hacer sus sumatorias.

- Conocimientos sociales, históricos, antropológicos. De su forma de vivir y conociendo su población e historia manejan su propia matemática.

- Conocimientos de historia, principios filosóficos y actividades educativas, sobre formación de culturas matemáticas viene hacer el último aprendizaje intercultural a través de los tiempos.

Según Aroca (2013) trabaja bajo dos campos, el rural y el urbano dentro de su investigación de la etnomatemática. Demostrando que cada campo muestra escenarios diferentes y cada uno aporta ideas justa para el uso de la matemática. En nuestro país tenemos estudiantes de diferentes partes del Perú. Muchos procedentes del campo rural donde vienen con conocimiento diferentes a la ciudad y la mezcla hacen un aprendizaje significativo de la matemática llevándolo a formarse usando su propio conocimiento y creando una nueva metodología de vida universitaria, trabajando y estudiando.

Considerando a Blanco-Álvarez (2006) (2011) como Aroca (2013) muestran que para el uso de las matemática y etnomatemática, necesitan del uso de la pedagogía y una adecuada 
metodología, logrando tener un pensamientos interculturales de mucha ayuda para estudiantes de diferentes culturas, y de esta forma logran desarrollar actividades matemáticas transculturales las cuales se van incorporando a la universidad y luego a la vida social. Y con la propuesta de Coll Us y Tec Chan (2018) orientada a la sensibilización sociocultural mediante la aplicación de la etnomatemática en el proceso de enseñanza aprendizaje de los estudiantes que serán futuros educadores desde su formación docente inicial y continua, se les desarrolla la habilidad para caracterizar el entorno y desenvolverse en zonas rural, teniendo en cuenta las disimiles condiciones en relación con el contexto urbano, teniendo en cuenta el diseño de actividades didácticas, las mismas que deben responder a los trabajos primarios, como lo son: la agricultura, pesca, ganadería, entre otros.

Autores como Bishop (1999) (2005), Gerdes (1999) (2007) y Oliveras (1996), mencionan que las investigaciones en etnomatemática, no siempre son del corte etnográfico, también de otros aprendizajes sociales y culturales de cada comunidad y en su recopilación de datos logran crear un nuevo algoritmo matemático que será y es de ayuda, por tanto cada aplicación de las etnomatemáticas solo cumplen un rol de mostrarse hacia su uso por una necesidad de supervivencia en lugares que no tienen una definición de la matemática. Para Albanese y Paredes (2014) lo vivenciado en el contexto y el entorno influyen poderosamente en las expresiones culturales y de ello no escapa la etnomatematica, constituye una de las representaciones enactivas e icónicas y del lenguaje simbólico, constituyendo modos de comunicación que permiten compartir formas de pensar, las cuales pueden ser descritas e interpretadas a través de investigaciones de corte etnográfico, dichas investigaciones logran recopilar aprendizajes sociales y culturales de cada comunidad y en su recopilación de datos al interpretarlos crean un nuevo algoritmo matemático.

Blanco Álvarez (2008) dice que el profesor D'Ambrosio ofrece métodos para desarrollar la etnomatemática, que incluye practicas a través de la observación a grupos naturales diferenciados, reconociendo sus acciones, a través de prácticas narrativas y finalizando con el análisis del discurso. Posteriormente afirma Blanco-Álvarez (2017) la etnomatemática constituye un programa de investigación en historia y filosofía de la matemática, articulada a la pedagogía, en el que cada integrante de la comunidad identifica su realidad natural a partir de experiencias y pensamientos, los cuales al ser históricamente desarrollados constituyen la cultura. Por ello, a la Etnomatemática le interesa investigar sobre los procesos de generación y difusión del conocimiento matemático. A manera de aporte Palacio (2019) afirma: "que el cálculo mental se da después de una comprensión significativa de cada una de las operaciones y sus propiedades, en cambio el algoritmo escrito enseñado por la escuela, solo permite generar una mecanización del procedimiento". Y finalmente el aporte del profesor D'Ambrosio (1985) : afirma: citado en "... el carpintero definitivamente trabaja con una idea matemática; los matemáticos que [deciden arbitrariamente dividir el ángulo usando solo una regla y una brújula] abordan la idea importante, y aunque son diferentes, están conectados por una idea"

La síntesis de la etnomatemática proviene de la acumulación de conocimientos matemáticos, prácticos y teóricos creados por grupos sociales en los que se asimilan en un contexto sociocultural diferente al primero, lo que ayuda a comprender el uso correcto de las matemáticas. 
Para el desarrollo de las matemáticas nacientes o primitivas, un individuo desarrolla muchos algoritmos etnomatemáticos: comprar, vender, existir en la vida. Con la única misión de ahorrar, reducir costos o administrar su dinero, y esto lo lleva al hecho de que aplica la etnomatemática utilizando algoritmos y, sin saber que está involucrado en las matemáticas, solo ellos tienen su propia forma de aplicarlo, porque en su proceso, desarrolla sumas, restas, clasifica, clasifica, calcula, mide y se organiza de acuerdo con el espacio y el tiempo, lo que considera elemental en su vida.

Finalmente Bartón, (1997) manifiesta que el uso de la etnomatemática, es darle valor a como el ser humano siempre ha tenido conocimiento de las matemáticas, solo que tienen su propio estilo étnico, y nuestro país cosmopolita tiene una variedad de culturas sociales y formas de vivir, las cuales son llevadas por los hijos a la universidad y ahí también es aplicada, lo cual bien llevada tendrá el mismo éxito de antes y esto enseña a la personas a crear ingresos adicionales por sus propios medios, y simplificar mucha veces su vida en la Universidad. En tanto, Osorio (2018) propone que la etnomatematica debe partir de las cosmovisiones y cosmogonías que manifiestan los habitantes de los pueblos y se debe entender como uno de los pilares fundamentales que no solo contribuye a desarrollar la matemática como ciencia, sino que forma parte del devenir histórico y apunta hacia el futuro. Por ello la Etnomatemática permite a ver la producción de conocimientos matemáticos, en función la variable sociocultural que sustenta el pensamiento y conocimiento ancestral.

Todo lo que muestra un país sobre la aplicación de sus sumas, restas, gráficos, orden, etc. en las calles, siendo útil para vivir, se simplifica a que usan etnomatemática, y puede generar ingresos y vivir tranquilamente.

El artículo muestra los resultados de hechos como los universitarios suelen aplicar esto algoritmos entomatemáticos, dentro su vida diaria y la complementa con sus estudios, y se logra lo que somos hoy en día. La importancia al estudio de la etmnomatemática es entender mejor las culturas de todo nuestro país y es de gran utilidad mostrar lo que somos sin perder nuestra cultura, la cual existe de muchas generaciones.

Un estudiante con valor hacia sus raíces e identidad valorativa, es un hombre de valor, y nuestra sociedad necesita de estas personas para que retornen a sus orígenes y vuelque los nuevos aprendizajes y haga de bien su comunidad. La universidad tiene que formar profesionales para el mundo, para fortalecer sus raíces, y entendiendo su cultura de vida es valorar más su identidad.

\section{Materiales y métodos}

El tipo de investigación es fundamental, dedicado a contribuir aportes de un tema específico a partir del enfoque cuantitativo, según; Hernández et. al (2014). Cuyo objetivo general es determinar los efectos de los algoritmos etnomatemáticos en el aprendizaje significativo de la matemática en estudiantes universitarios, en el año 2019. Considerando 3 objetivos específicos para sintetizar mejor el estudio siendo los siguientes; analizar las operaciones básicas que se desarrollan al aplicar algoritmos etnomatemáticos en la vida cotidiana

Esta obra se comparte bajo la licencia Creative Common Atribución-No Comercial 4.0 International (CC BY-NC 4.0) Revista de la Universidad Internacional del Ecuador. URL: https://www.uide.edu.ec/ 
propician el conflicto cognitivo en estudiantes; determinar si las situaciones más comunes en las que se emplean algoritmos etnomatemáticos facilitan el aprendizaje de relaciones binarias en estudiantes universitarios en el año 2019; establecer si el uso de algoritmos etnomatemáticos mejora el aprendizaje de proposiciones.

La investigación se realizó a nivel explicativo la misma está encaminada a explicar el efecto del fenómeno detallado en su realidad social. Adoptando un diseño cuasi experimental con una sección transversal con dos etapas de evaluación: una prueba preliminar y una prueba posterior con un grupo de investigación.Galeano (2004) La población está compuesta por estudiantes universitarios s de diferentes universidades, siendo de 3 universidades privadas (Wiener, UTP y UPN) y 2 universidades Publicas (UNI, UNMSM), sumando una población total de 60; y con una muestra de 25 estudiantes universitarios seleccionados usando una muestra probabilística deliberada, ya que fue tomada como una guía para estudiantes con un promedio de edad, entre 18 y 25 años, entre hombres y mujeres. Para recopilar datos, se utilizó una técnica de observación para identificar el problema en sí, para determinar específicamente qué tipo de algoritmos usan en el mismo ámbito. Y una entrevista escrita que se aplicó a la muestra utilizando algoritmos etnomatemáticos. Y como instrumentos se usó una lista de cotejos conteniendo dimensiones e indicadores requeridos en la recolección e algoritmos etnomatematicos. Y finalmente una ficha bibliográfica con el fin de registrar información analizada para contribuir los fundamentos teóricos de las variables y la metodología de la investigación.

Para procesar los datos obtenidos, se clasificaron según una escala ordinal e intervalo. Se usó una escala ordinal para derivar los puntajes pre y post prueba. Y la escala de intervalos se utilizará para ubicar los puntajes finales en las categorías de intervalos y aplicar estadísticas de casos; se consideraron los intervalos de 0 a 4 correspondiéndole la categoría "Muy bajo"; de 4 a 8 "Bajo"; de 8 a 12 "Regular"; de12 a 16 "Alto" y de "16 a 20" Muy alto". Ríos. (1983) Finalmente, los datos se tabularon con los resultados obtenidos al calificar y posicionar en las categorías de intervalos provistos para el análisis estadístico. Estos datos numéricos obtenidos del recuento se ubicarán en las tablas de asignación de frecuencias, y para la estadística inferencial se ha utilizado la prueba de significación estadística la "T" de Student para muestras desiguales menores que 30 y lo comprobaremos con los datos obtenidos en el pret test que se aplicó al grupo experimental para determinar el grado de desarrollo del aprendizaje significativo, una vez calculado el valor se verificó si cae en la región de aceptación o rechazo de la hipótesis nula, adoptando una decisión al respecto. primeramente se aplicó el Pre Test, dicha prueba consistió en aplicar en la actividad laboral, donde se estableció 6 sesiones de trabajo destinadas al desarrollo de su capacidad lógico matemática; mientras que en la lista de cotejo se planteó 10 indicadores considerándose los criterios SI - NO y finalmente el cuestionario que se aplicó tanto a los estudiantes universitarios del grupo experimental con la finalidad de recoger información sobre el desarrollo de las capacidades en el manejo de algoritmos lógico matemáticos. Y finalmente el post test tales como: cuestionario, lista de cotejo; instrumentos que fueron aplicados con la finalidad de poder determinar el mejoramiento de las capacidades investigadas en relación al aprendizaje significativo después de la aplicación de los algoritmos etnomatemáticos. 


\section{Resultados}

La aplicación de algoritmos etnomatemáticos como estrategia para lograr el aprendizaje significativo en estudiantes de educación superior universitaria en la ciudad de Lima- año 2019, se trabajó con una muestra experimental de 25 estudiantes a quienes se les aplicó un cuestionario sobre aprendizaje significativo de la etnomatemática a modo de pre test y post test; en la aplicación del pre test, tal como figura en la Tabla $\mathrm{N}^{\circ} 01$, se obtuvo que 1 estudiante se situó en un 4\% ubicándose en la categoría "Muy bajo", 9 estudiantes que representan el 36\% se ubicaron en la categoría "Regular". Asimismo 11 estudiantes universitarios, con un 44\% alcanzaron la categoría "Alto" finalmente 4 estudiantes universitarios con un 16\% se coloca como "Muy alto", ningún estudiantes se ubicó en la categoría "Bajo"; en la fase post test, aplicando el mismo cuestionario se obtuvieron resultados que posicionan con 2 estudiantes universitarios con un $8 \%$ a los evaluados en la categoría "Muy bajo"; Así mismo 3 estudiantes universitarios con un $12 \%$ se colocan en la categoría "Bajo"; y 5 estudiantes universitarios con el 20\% se posicionó como "Regular"; Y con 9 estudiantes universitarios con el 36\% se situó como "Alto" finalmente 6 estudiantes universitarios con el 24\% alcanzaron la categoría de "Muy alto". Al contrastar la media aritmética, tanto en la prueba pre test y post test, se obtuvieron para la prueba pre test un valor de media de 8,36 y en la prueba post test la media fue de 16,12, habiéndose incrementado en 7,76 puntos lo cual implica que la aplicación de algoritmos ha sido eficaz.

Los resultados se respaldan en la prueba de la primera hipótesis operacional que arroja un valor estadístico de un $\mathrm{T}=1,02$, permite apreciar que las operaciones básicas subsumidas en el algoritmo etnomatemático permiten el conflicto cognitivo, por ejemplo al pagar un servicio con billetes se debe establecer mentalmente si el comerciante para dar vuelto, resta o completa el valor de acuerdo al billete o monedas con las cuales se cancela; un valor de $\mathrm{T}=0,09$ se refleja en la tercera hipótesis operacional, lo que ubica dicha valor en la región de aceptación mostrando la efectividad de la aplicación de dichos algoritmos para el aprendizaje de proposiciones tales como "el precio de un x producto es caro, no puedo comprarlo hasta que baje de precio, por lo tanto debo sustituirlo por otro". En lo referente a la segunda hipótesis operacional se tiene que el $88 \%$ afirma que las situaciones de índole comercial son las que más permiten utilizar algoritmos etnomatemáticos, esta es la de comprar lo que facilita el establecimiento de relaciones binarias, al relacionar cantidades con precios, lo percibido por un día de trabajo con lo gastado, entre otros.

Se concluye que, el grupo experimental, constituido por estudiantes universitarios que fueron sometidos a la aplicación frecuente de algoritmos etnomatemáticos en las sesiones de aprendizaje de la asignatura de matemática, ha logrado incrementar significativamente su aprendizaje. 


\section{Tabla 1}

Resultados del pre-test del grupo experimental sobre el calificativo obtenido en el nivel de aprendizaje significativo de la etnomatemática.

\begin{tabular}{|c|c|c|c|c|c|c|c|c|c|c|c|c|}
\hline \multicolumn{13}{|c|}{ PRE - TEST } \\
\hline \multicolumn{4}{|c|}{ INTERVALO } & \multirow{2}{*}{$\frac{\mathbf{f i}}{1}$} & \multirow{2}{*}{$\frac{\text { mcl }}{3}$} & \multirow{2}{*}{$\frac{\text { f.mcl }}{3}$} & \multirow{2}{*}{$\frac{\overline{\mathbf{X}}}{8,36}$} & \multirow{2}{*}{$\begin{array}{c}\mathbf{d} \\
-5,36\end{array}$} & \multirow{2}{*}{$\frac{\mathbf{D}^{2}}{28,73}$} & \multirow{2}{*}{$\begin{array}{l}\text { F.d }^{2} \\
28,73\end{array}$} & \multirow{2}{*}{$\begin{array}{c}\text { DS } \\
1,76\end{array}$} & \multirow{2}{*}{$\frac{\text { Fi } \%}{4}$} \\
\hline[ & 0 & 4 & $>$ & & & & & & & & & \\
\hline[ & 4 & 8 & $>$ & 0 & 5 & 0 & 8,36 & $-3,36$ & 11,29 & 0 & 1,76 & 0 \\
\hline[ & 8 & 12 & $>$ & 9 & 7 & 63 & 8,36 & $-1,36$ & 1,85 & 16,65 & 1,76 & 36 \\
\hline [ & 12 & 16 & $>$ & 11 & 9 & 99 & 8,36 & 0,64 & 0,41 & 4,51 & 1,76 & 44 \\
\hline [ & 16 & 20 & ] & 4 & 11 & 44 & 8,36 & 2,64 & 6,97 & 27,88 & 1,76 & 16 \\
\hline & TAL & & & 25 & & 209 & & & & 77,77 & & 100 \\
\hline
\end{tabular}

Fuente: Elaboración propia

\section{Tabla 2}

Resultados del post test del grupo experimental sobre el calificativo obtenido en el nivel de aprendizaje significativo de la etnomatemática.

\begin{tabular}{|c|c|c|c|c|c|c|c|c|c|c|c|c|}
\hline \multicolumn{13}{|c|}{ POST - TEST } \\
\hline \multicolumn{4}{|c|}{ INTERVALO } & \multirow{2}{*}{$\begin{array}{r}\mathbf{f i} \\
2\end{array}$} & \multirow{2}{*}{$\begin{array}{r}\text { mcl } \\
11\end{array}$} & \multirow{2}{*}{$\begin{array}{c}\text { f.mcl } \\
22\end{array}$} & \multirow{2}{*}{$\begin{array}{c}\overline{-} \\
\mathbf{X}\end{array}$} & \multirow{2}{*}{$\begin{array}{c}\text { d } \\
-5,12\end{array}$} & \multirow{2}{*}{$\begin{array}{c}\mathbf{d}^{2} \\
26,21\end{array}$} & \multirow{2}{*}{$\begin{array}{l}\text { F.d }^{2} \\
52,42\end{array}$} & \multirow{2}{*}{$\begin{array}{c}\text { DS } \\
2,4\end{array}$} & \multirow{2}{*}{$\begin{array}{c}\text { Fi\% } \\
8\end{array}$} \\
\hline[ & 0 & & $4>$ & & & & & & & & & \\
\hline[ & 4 & & $8>$ & 3 & 13 & 39 & 16,12 & $-3,12$ & 9,7 & 29,2 & 2,4 & 12 \\
\hline[ & 8 & 1 & $2>$ & 5 & 15 & 75 & 16,12 & $-1,12$ & 1,25 & 6,3 & 2,4 & 20 \\
\hline [ & 12 & 1 & $6>$ & 9 & 17 & 153 & 16,12 & 0,88 & 0,77 & 6,93 & 2,4 & 36 \\
\hline [ & 16 & 2 & 0 ] & 6 & 19 & 114 & 16,12 & 2,88 & 8,29 & 49,74 & 2,4 & 24 \\
\hline \multicolumn{4}{|c|}{ TOTAL } & 25 & & 403 & & & & 144,54 & & 100 \\
\hline
\end{tabular}

Fuente: Elaboración propia

\section{La prueba de las hipótesis}

\section{Hipótesis general}

H1: La frecuencia de la aplicación de algoritmos etnomatemáticos en las sesiones de aprendizaje, incrementa el aprendizaje significativo de la matemática, en estudiantes universitarios en el año 2019.

\section{Hipótesis Operacional}


H2: Las operaciones básicas desarrolladas mediante algoritmos etnomatemáticos propician el conflicto cognitivo en estudiantes universitarios en el año 2019.

H3. Las situaciones más comunes en las que se emplean algoritmos etnomatemáticos son las relacionadas a la actividad comercial los que facilitan el aprendizaje de relaciones binarias en estudiantes universitarios en el año 2019.

H4. El uso de algoritmos etnomatemáticos mejora el aprendizaje de proposiciones facilitando la comprensión de la lógica matemática en estudiantes universitarios en el año 2019.

\section{La hipótesis operacional "A" queda planteada de la siguiente manera:}

Las operaciones básicas más utilizadas en los algoritmos etnomatemáticos propician el desarrollo del conflicto cognitivo

Para verificar esta hipótesis es necesario utilizar la prueba de significación estadística " $T$ " de Student para muestras desiguales menores que 30 y lo comprobaremos con los datos obtenidos en el Pre - test aplicado al grupo experimental, con la finalidad de determinar el grado de desarrollo del aprendizaje significativo, antes de la aplicación de los métodos algorítmicos etnomatemáticos; para ello planteamos las siguientes hipótesis.

\section{Planteamiento de la hipótesis:}

Ho: Las operaciones básicas más utilizadas en los algoritmos etnomatemáticos no propician el desarrollo del conflicto cognitivo

Hi: Las operaciones básicas más utilizadas en los algoritmos etnomatemáticos propician el desarrollo del conflicto cognitivo

\section{Fórmula:}

$$
\mathbf{T}=\frac{\mid \mathbf{X e 1}-\mathbf{X e} 2 /}{\left(\sqrt{\frac{(n 1-1) s 1+(n 2-1) s 2}{n 1+n 2-2}}\right)\left(\sqrt{\frac{n 1+n 2}{n 1 \cdot n 2}}\right)}
$$

Donde:

Xe 1= Media Aritmética del grupo experimental pre test

Xe2 = Media Aritmética del grupo experimental post test

S1 = Desviación Estándar del grupo experimental fase pre test

S2 = Desviación Estándar del grupo experimental fase post test

$\mathbf{n} \mathbf{1 = n 2}=$ Tamaño de la muestra 
$/ 8.36-7.75 /$

$\mathbf{T}=$

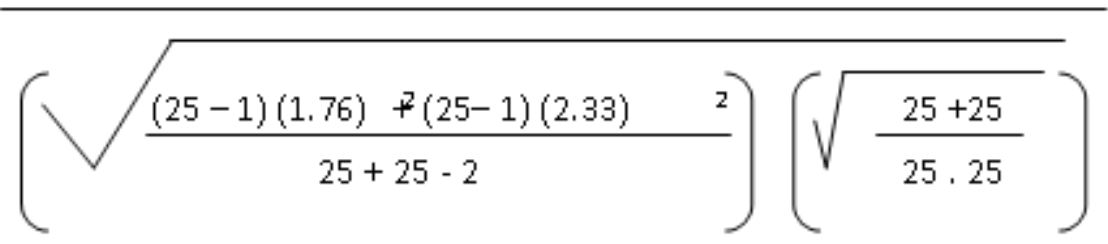

$\mathbf{T}=1,02$

\section{Esquema Gráfico:}

Determinando el valor crítico:

$\mathbf{V}=$ Grado de libertad

$$
\mathrm{V}=\mathrm{n} 1+\mathrm{n} 2-2
$$

Siendo $\mathrm{n} 1+\mathrm{n} 2=50$

$\mathrm{V}=25+25-2$

$\mathrm{V}=50-2$

$\mathrm{V}=48^{\circ}$ de libertad

En la tabla $\mathrm{TC}=2.02$

En la tabla $\mathrm{TC}=2.02$

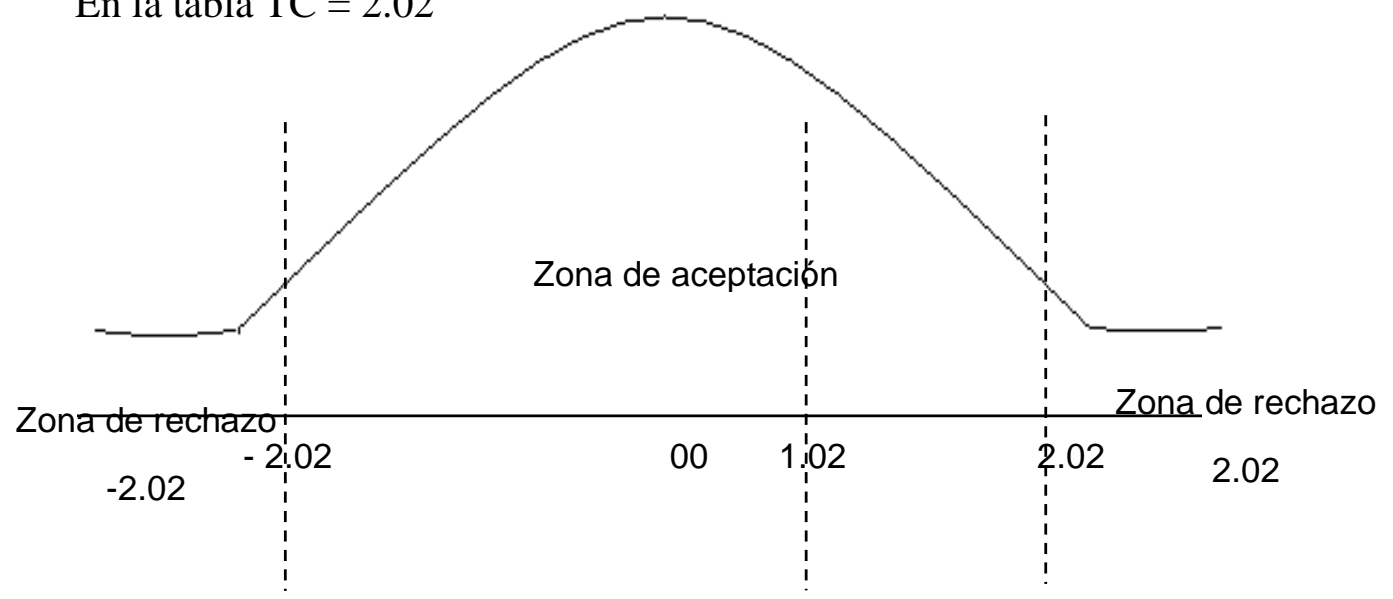

Conclusión: Según los resultados obtenidos por el estadígrafo $\mathrm{T}=1.02$ el cual se ubica en la región de aceptación por lo cual aceptamos Hi y rechazamos Ho, por lo tanto se comprueba que las operaciones básicas más utilizadas en los algoritmos etnomatemáticos propician el desarrollo del conflicto cognitivo.

\section{La hipótesis operacional "B" se plantea de la siguiente manera:}

Las situaciones más comunes en las que se emplean algoritmos etnomatemáticos son las relacionadas a la actividad comercial los que facilitan el aprendizaje de relaciones binarias matemáticas por parte de los estudiantes universitarios 


\section{Tabla 3}

¿Cuál de las siguientes operaciones en base a la etnomatemáticos consideras que mejora tu capacidad de establecer relaciones binarias?

\begin{tabular}{lll}
\hline CRITERIOS & Fi & $\mathbf{f \%}$ \\
\hline Comprando & 22 & 88 \\
Vendiendo & 3 & 12 \\
Mirando & 0 & 0 \\
N.A & 0 & 0 \\
T.A & 0 & 0 \\
TOTAL & 25 & 100 \\
\hline
\end{tabular}

Fuente: Elaboración Propia

\section{Figura 1}

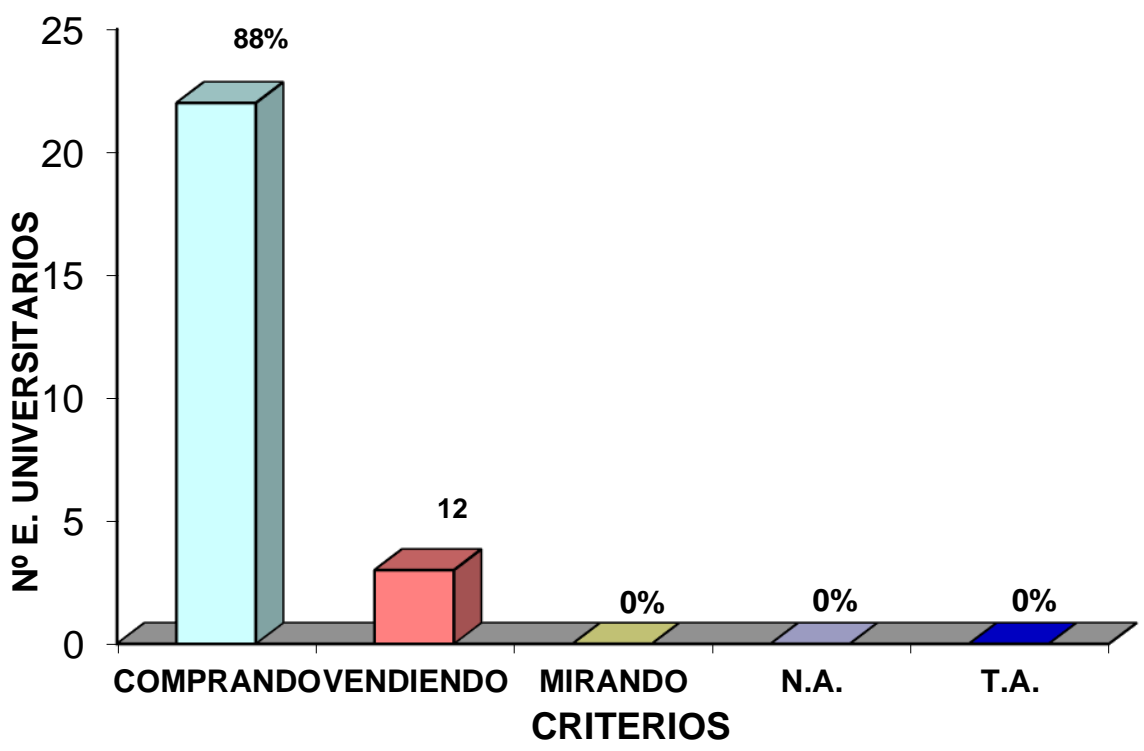

Para verificar esta hipótesis es necesario recurrir a los resultados obtenidos del cuadro $\mathrm{N}^{\mathrm{o}}$ 3 del cuestionario, el cual afirma que: de un total de 25 estudiantes universitarios, que representan el 100\%, 22 estudiantes universitarios manifiestan que el método etnomatemático de realizar compras ayudó a mejorar su capacidad de realizar operaciones binarias matemáticas básicas, representando el $88 \%$, mientras que 3 estudiantes universitarios manifestaron que el método de venta contribuye a mejorar su capacidad de realizar operaciones matemáticas, representando el $12 \%$. 
Conclusión: Llegando a comprender que la mayoría de los estudiantes universitarios del grupo experimental manifiestan que el método de realizar compras simuladas ayudó a mejorar su capacidad de realizar operaciones matemáticas básicas, debido a que conocen la importancia que tienen estos métodos en el desarrollo de habilidades matemáticas. Por lo tanto se concluye que: Las situaciones más comunes en las que se emplean algoritmos etnomatemáticos son aquellas en las que las personas realizan intercambio comercial (compra-venta) facilitando el aprendizaje de relaciones binarias. Quedando verificada la hipótesis operacional " $\mathrm{B}$ "

\section{La hipótesis operacional "C" queda planteada de la siguiente manera:}

El uso de algoritmos etnomatemáticos mejora el aprendizaje de proposiciones facilitando la comprensión de la lógica matemática.

Para verificar esta hipótesis es necesario utilizar la prueba de significación estadística "T "de Student, para muestras desiguales menores que 30 y lo comprobaremos con los datos obtenidos en el post - test, aplicado a los alumnos del grupo experimental y de control, con la finalidad de determinar si existe diferencia significativa en el nivel de desarrollo de las capacidades básicas entre ambos grupos, después de la aplicación de los métodos adecuados; para ello planteamos las siguientes hipótesis :

\section{Planteamiento de Hipótesis}

Ho: El uso de algoritmos etnomatemáticos no mejora el aprendizaje de proposiciones facilitando la comprensión de la lógica matemática

Hi: El uso de algoritmos etnomatemáticos mejora el aprendizaje de proposiciones facilitando la comprensión de la lógica matemática.

Usaremos la prueba de significación estadística "T" de Student, extrayendo los datos de la media aritmética, la desviación estándar del post test y la cantidad de estudiantes universitarios del grupo experimental, empleando para ello la siguiente fórmula.

\section{Fórmula:}

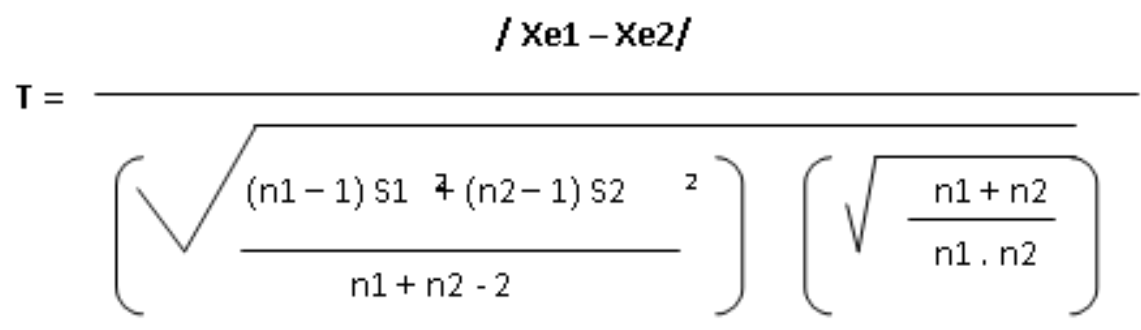

Donde:

Xe1 = Media Aritmética del grupo experimental etapa pre test

Xe 2= Media Aritmética del grupo experimental etapa post test

S1 = Desviación Estándar de la Prueba pre test

S2 = Desviación Estándar de la prueba post test

$\mathbf{n} \mathbf{1}=\mathbf{n} \mathbf{2}=$ Tamaño de las muestras 
$/ 16.12-8.32 /$

$\mathbf{T}=$

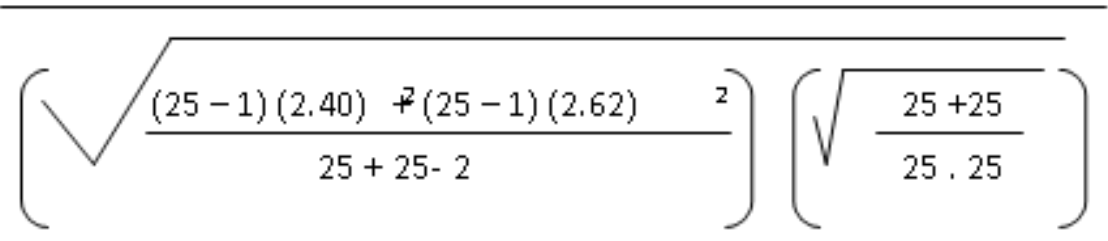

$\mathbf{T}=0.09$

\section{Esquema Grafico:}

\section{Determinando el valor crítico:}

$\mathbf{V}=$ Grado de libertad

$$
\mathrm{V}=\mathrm{n} 1+\mathrm{n} 2-2
$$

Siendo $\mathrm{n} 1+\mathrm{n} 2=50$

$$
\begin{aligned}
& \mathrm{V}=25+25-2 \\
& \mathrm{~V}=48-2 \\
& \mathrm{~V}=48^{\circ} \text { grados de libertad }
\end{aligned}
$$

En la tabla $\mathrm{TC}=2.02$

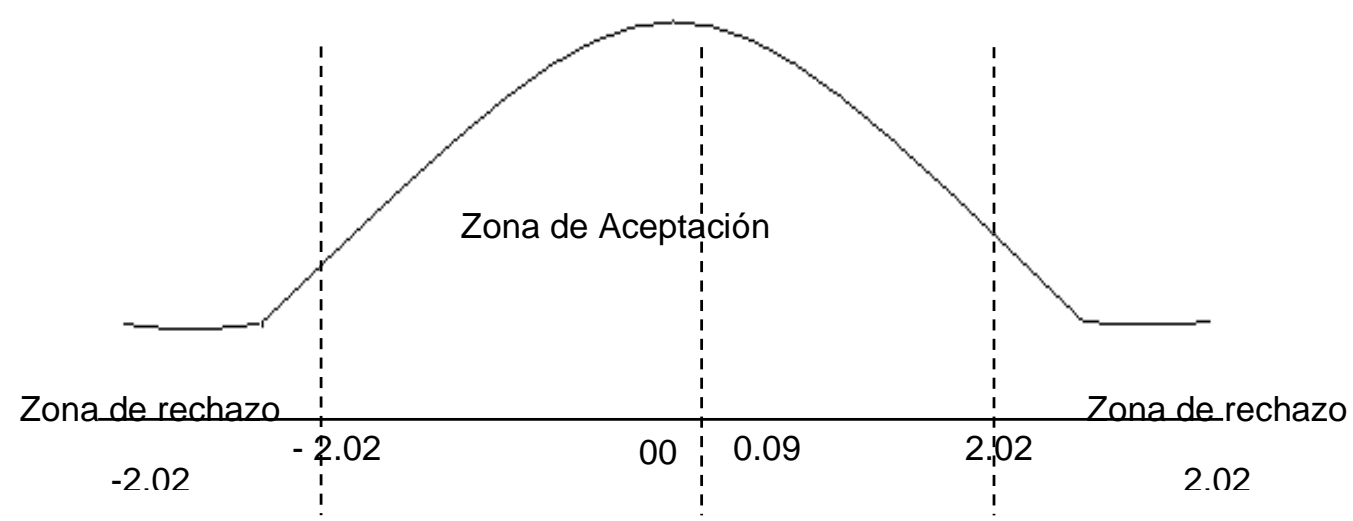

Conclusión: Según los resultados obtenidos en el estadígrafo $\mathrm{T}=0.09$ el cual se ubica en la región de aceptación por lo cual aceptamos Hi y rechazamos Ho, por lo tanto, se comprueba que la aplicación de los algoritmos etnomatemáticos si permite el desarrollo del aprendizaje de proposiciones facilitando la comprensión de la lógica matemática.

\section{La hipótesis general queda planteada de la siguiente manera:}

Cuanto más frecuente es el uso de los algoritmos etnomatemáticos más repercute en el aprendizaje significativo de la matemática de los estudiantes universitarios. 
La verificación de la hipótesis operacional " $A$ ” de acuerdo a los resultados obtenidos por el estadígrafo $\mathrm{T}=1.02$ el cual se ubica en la región de aceptación por lo cual aceptamos Hi y rechazamos Ho, por lo tanto se comprueba que las operaciones básicas más utilizadas en los algoritmos etnomatemáticos propician el desarrollo del conflicto cognitivo.

La verificación de la hipótesis operacional " $B$ ” comprueba que los estudiantes universitarios del grupo experimental mejoraron el aprendizaje de relaciones binarias matemáticas por parte de los estudiantes universitarios; lo que más repercutió en el aprendizaje significativo de la matemática por parte de ellos. Quedando verificada la hipótesis operacional "B"

La verificación de la hipótesis operacional “ $C$ ” comprueba que según los resultados obtenidos en el estadígrafo $\mathrm{T}=0,09$ el cual se ubica en la región de aceptación por lo cual aceptamos Hi y rechazamos Ho, por lo tanto se comprueba que la aplicación de los algoritmos etnomatemáticos si permite el desarrollo del aprendizaje de proposiciones facilitando la comprensión de la lógica matemática.

Conclusión final: Por lo tanto, queda comprobado estadísticamente que cuanto más frecuente es el uso de los algoritmos etnomatemáticos más repercute en el aprendizaje significativo de la matemática de los estudiantes universitarios.

\section{Discusión de resultados}

La verificación de la hipótesis operacional " $A$ ” de acuerdo a los resultados obtenidos por el estadígrafo $\mathrm{T}=1.02$ el cual se ubica en la región de aceptación por lo cual aceptamos Hi y rechazamos Ho, por lo tanto se comprueba que las operaciones básicas más utilizadas en los algoritmos etnomatemáticos propician el desarrollo del conflicto cognitivo.

Teniendo en cuenta que el conflicto cognitivo constituye la contradicción entre los conocimientos que ya se poseen con nuevos conocimientos, para el caso, acerca de la matemática, tomando como punto de partida los algoritmos que se producen a partir de la cotidianeidad social, y que la prueba de hipótesis arroja que efectivamente el manejo de los citados algoritmos propicia el desarrollo cognitivo, se tendría que este se produce a raíz de la obtención del algoritmo es una abstracción de una actividad comercial cotidiana, ello le da una sólida base que parte del desarrollo de la ciencia matemática formal y lógica con aquella matemática del diario vivir, práctica y que se ha desarrollado en la pura interactividad.

La verificación de la hipótesis operacional “ $B$ ”, obtuvo un $\mathrm{T}=0,09$ comprueba que los estudiantes universitarios del grupo experimental mejoraron debido a la frecuencia del uso de los algoritmos etnomatemáticos desarrollando sus conocimientos sobre relaciones binarias ello fue un detonante para el aprendizaje significativo de la matemática lo que verifica la hipótesis operacional "B".

La forma como se desarrollan las relaciones binarias son en pares ordenados, cuando alguien acude a realizar alguna compra se relaciona el producto con el precio, ello constituye una 
relación binaria, lo que constituye un punto de partida para desarrollar funciones y posteriormente todo un engranaje matemático hasta el análisis infinitesimal.

La verificación de la hipótesis operacional “ $C$ ” comprueba que según los resultados obtenidos en el estadígrafo $\mathrm{T}=0,09$ el cual se ubica en la región de aceptación por lo cual aceptamos Hi y rechazamos Ho, por lo tanto se comprueba que la aplicación de los algoritmos etnomatemáticos si permite el desarrollo del aprendizaje de proposiciones facilitando la comprensión de la lógica matemática.

Por lo tanto, queda comprobado estadísticamente que cuanto más frecuente es el uso de los algoritmos etnomatemáticos incide en el aprendizaje significativo de la matemática de los estudiantes universitarios.

\section{Conclusiones}

La significatividad del aprendizaje de la matemática, en cuanto el actor social que para el caso es el estudiante universitario se verifica al comprobar las hipótesis planteadas, en relación al primera hipótesis operacional " $\mathrm{A}$ " se obtuvo un estadígrafo $\mathrm{T}=1.02$ ubicado en la región de aceptación con lo que se acepta Hi y rechaza Ho, lo cual demuestra que las operaciones básicas más utilizadas en los algoritmos etnomatemáticos propician el desarrollo del conflicto cognitivo, implicando que trasladar la cotidianidad matemática al aula de clases constituye una herramienta poderosa para el aprendizaje.

Con respecto a los resultados de la contrastación de la hipótesis operacional "B" se sitúa en un estadígrafo $\mathrm{T}=0,09$ lo cual también constituye un parámetro aceptable, la hipótesis operacional "B" establece que la frecuencia del uso de los algoritmos etnomatemáticos desarrolla conocimientos sobre relaciones binarias, tales como relacionar cantidad con precio; lo que se gana con lo que se gasta; lo que se consume con lo presupuestado; al comprobarse ello se tiene que la recolección de algoritmos etnomatemáticos genera que el estudiante construya una serie de relaciones binarias, las misma que pueden ser analizadas en el aula logrando abstraer la realidad a partir de una experiencia vivencial.

La hipótesis operacional "C" persigue demostrar que el desarrollo de la lógica también parte de la experiencia cotidiana y que el accionar de los actores, al realizar transacciones económicas, desarrolla dicho pensamiento, en tal sentido, la hipótesis se sustenta en el hecho de la aplicación de los algoritmos etnomatemáticos en el desarrollo del aprendizaje de proposiciones facilitando la comprensión de la lógica matemática, lo cual al ser sometido al análisis estadístico arrojo un $\mathrm{T}=0,9$ similar al resultado con la hipótesis operacional " $\mathrm{B}$ ", lo cual confirma que el acto de realizar una transacción comercial sigue una estructura lógica que al ser reproducida al interior del aula adopta la forma de un algoritmo. Así, por ejemplo, formando silogismos tales como: si, gasto todo mi dinero, no tendré para comprar mis cuadernos; me dio hambre y gaste toda mi propina en comida, luego no poder adquirir mis cuadernos.

Como conclusión se tiene que si se han comprobado las hipótesis operacionales queda comprobada la hipótesis general, la misma que tarta sobre la frecuencia de la aplicación de 
algoritmos etnomatemáticos en las sesiones de aprendizaje la misma que incide en el aprendizaje significativo de la matemática, en estudiantes universitarios en el periodo 2019, contrastación que se hizo a partir del desarrollo cognitivo del estudiante asociado a la abstracción, a partir de la realidad social, de relaciones binarias y lógica.

\section{Referencias Bibliográficas}

Albanese , V., \& Perales , F. (2014). Pensar matemáticamente: una visión etnomatemática de la práctica artesanal soguera. Latinoamericana de Investigación en Matemática Educativa, 17(3), 261-288. doi:http://dx.doi.org/10.12802/relime.13.1731

Aroca, A. (2013). Los escenarios de exploración en el Programa de Investigación en Etnomatemáticas. Revista Educación Matemática, 25(7), 111-131.

Aroca, A. (2016). La definición etimológica de Etnomatemática e implicaciones en Educación Matemática. SciELO Analytics, 28(2), 175-195. Obtenido de http://www.scielo.org.mx/scielo.php?pid=S1665-

$58262016000200175 \&$ script $=$ sci_abstract

Ascher, M. (1982). Etnomathemics. History of science, 24(01), 15-18.

Ascher, M., \& D'Ambrosio, U. (1994). Ethnomathematics: A dialogue. For the Learning of Mathematics, 14(02), 36-43.

Bartón, B. (1997). Teniendo el Sentido de la Etnomatemática: New Zeland.: The University of Auckland.

Bishop, A. (1999). Enculturación matemática: la educación matemática desde una perspectiva cultural. Barcelona: Paidós.

Bishop, A. (2005). Aproximación sociocultural a la educación matemática. Santiago de Cali: Universidad de Valle.

Blanco-Álvarez, H. (2006). La Etnomatemática en Colombia. Un programa en construcción. Revista Bolema: Boletim de Educação Matemática,19(26), 49-75.

Blanco-Álvarez, H. (2008). Entrevista al profesor Ubiratan D'Ambrosio. Revista Bolema: Boletim de Educação Matemática, 19(26), , 49-75.

Blanco-Álvarez, H. (2011). La postura sociocultural de la educación matemática y sus implicaciones en la escuela. Revista Educación y Pedagogía, 23(59), , 59-66.

Blanco-Álvarez, H. (2017). Elementos para la formación de maestros de matemáticas desde la etnomatemática. Tesis Doctoral. Universidad de Granada, Colombia.

Bonilla, M., Rosa , M., Auccahuallpa , R., \& Reyes , M. (2018). La dimensión matemática en educación intercultural bilingüe: educación matemática y diversidad. 31, pág. 8. Brasil: Acta Latinoamericana de Matemática Educativa. Obtenido de http://www.repositorio.ufop.br/handle/123456789/11403

Cahuana,, Á., Salinas , R., \& Ruiz , E. (2018). Influencia etnomatemática en la resolución de problemas en estudiantes del primer grado de la institución educativa bilingüe san francisco distrito Yarinacocha. Tzhoecoen, 24(3), 45-55.

Collí Us, S., \& Tec Chan , P. (2018). Matemáticas para todos. Investigación e Innovación en Matemática Educativa, 3, 140-143. Obtenido de http://revistaiime.org/index.php/IIME

D’Ambrosio, U. (2001). Paz, Educacao Matemática e Etnomatematica. En terori e Practica da Educacao. Brasil: Maringá, PR.

Esta obra se comparte bajo la licencia Creative Common Atribución-No Comercial 4.0 International (CC BY-NC 4.0) Revista de la Universidad Internacional del Ecuador. URL: https://www.uide.edu.ec/ 
D’Ambrosio, U. (2002). Etnomatemática, entre las tradiciones y la modernidad. Belo Orizonte: Autentica.

D'Ambrosio, U. (1985). Ethnomathematics and its place in the history and pedagogy of mathematics. EEUU: For the Learning of Mathematics.

Dos Santos Bernardi , L., \& Santos , J. (2018). Etnomatemática y Pedagogía Freireana: una experiencia intercultural con la comunidad Kaingang. Zetetike, 26(1), 147-166. doi:https://doi.org/ 10.20396/zet.v26i1.8650727

Fuentes, C. (2019). Etnomatemática para comprender la realidad: analizando la calidad de vida en algunos países de Latinoamérica. Latinoamericana de Etnomatemática, 12(1), 25-43. Obtenido de http://funes.uniandes.edu.co/15344/1/Fuentes2019Etnomatematica.pdf

Galeano. (2004). Diseñosde proyectos en la investigación cualitativa. Medellin: Fondo Editorial.

Gerdes, P. (1989). The use of the etnomathematics in the classroom, procededings of politics of mathematics education conference,. NEE Mathematics Commission, University of westen cape, 26-36.

Gerdes, P. (1996). Ethnomathematics and Mathematics Education. En A. Bishop, K. Clements, C. Keitel, J. Kilpatrick, \& C. Laborde. International Handbook of Mathematics Education. Dordrecht: Kluwer Academic Publishers., 909-943.

Gerdes, P. (1999). Geometry form Africa, Matehmatical and educational exploration. Moçambique: Nampula: Universidade Lúrio Nampula. .

Gerdes, P. (2007). Otthava, Fazer Cestos e Geometria na Cultura Makhuwa do Nordeste de Moçambique. Mozambique: Universida de Lúrio Nampula. Obtenido de http://stores.lulu.com/pgerdes

Gomez Gomez, J. E. (2019). Estado del arte de la etnomatemáticas en el Perú. Grado de Bachiller. Universidad Nacional Mayor de San Marcos, Lima. Obtenido de https://cybertesis.unmsm.edu.pe/bitstream/handle/20.500.12672/11758/Gomez_gj.pdf?se quence $=1 \&$ isAllowed $=\mathrm{y}$

Guzmán Marín, F. (2018). Los retos de la educación intercultural en el siglo XXI. Latinoamericana de educación inclusiva, 12(1), 199-212. doi: https://doi.org/10.4067/S0718-73782018000100013

Hernández Sampieri, R., Fernández Collado, C., \& Baptista Lucio, M. (2014). Metoodología de la Investigación (Sexta ed.). Mexico: McGRAW-HILL / INTERAMERICANA EDITORES, S.A. DE C.V.

Knijnik, G., Wanderer, F., Giongo, I., \& Duarte, C. (2012). Etnomatemática em movimento. Belo Horizonte : Autentica.

Miarka, R., \& Viggiani, M. A. (2012). Matemática e/na/ou Etnomatemática? Latinoamericana de Etnomatemática,, 5(01), 149-158.

Oliveras, M. (1996). Etnomatemáticas, formación de profesores e innovación curricular. Granada: Comares.

Osorio, C. T. (2018). Licenciatura en Pedagogía de la Madre Tierra, etnomatemática y formación $\begin{array}{lllll}\text { de profesores. Ciência \& Educação (Bauru), } & 10(1), \quad 759-777 .\end{array}$ doi:https://doi.org/10.1590/1516-731320180030014.

Palacio Palmera, R. J. (2019). Estudio etnomatemático sobre las estrategias de cálculo aritmético de comerciantes del mercado Baltazar R. Leyva Mancilla. Tesis de Maestría. Universidad Autónoma de Guerrero, Mexico.

Ríos. (1983). Análisis estadístico aplicado. Madrid: Paraninfo. 
Saca Ramos, M. V. (2019). Recursos didácticos de la sabiduría ancestral que fomenten la interculturalidad en la etnomatemática en los niños de la básica elemental. Grado de Maestría. Universidad Nacional del Chimborazo, Riobamba, Ecuador. Obtenido de http://dspace.unach.edu.ec/bitstream/51000/5313/1/UNACH-EC-IPG-PED-DOC-20190003.pdf

Santana, J. A. (2019). Práticas escolares para mobilização da cultura matemática de estudantes da eja por meio da etnomatemática. Mestrado profissional em educação de jovens e adultos mpeja. Universidade do estado da bahia-uneb, Estado de Bahia.

Valencia Salinas, P., \& Diaz Villaneda, E. (2019). Estrategia didáctica para fortalecer el Razonamiento Algebraico fundamentada en la Etnomatemática. Licenciatura en Matemáticas y Física. Universidad de Antioquia, Antioquia, Colombia. Obtenido de http://ayura.udea.edu.co:8080/jspui/bitstream/123456789/3533/1/ValenciaSalinasPaola DiazVillanedaEstefania_DidacticaRazonamientoAlgebraico_2019_TG.pdf

Villota , J., \& Singo , J. (2018). Inserción de la etnomatemática en la formación docente en la educación superior ecuatoriana. Anales, 1(376), 129-144.

Viteri Gordillo, M. (2015). La Etnomatemática en el sistema educativo ecuatoriano. Publicando, $\begin{array}{lll}2(2), & 24-34 . & \text { Obtenido }\end{array}$

https://revistapublicando.org/revista/index.php/crv/article/view/20 